\title{
Modeling Scramjet Flows with Variable Turbulent Prandtl and Schmidt Numbers
}

\author{
X. Xiao, H. A. Hassan ${ }^{\dagger}$ \\ North Carolina State University, Raleigh, NC 27695-7910 \\ R. A. Baurle \\ NASA Langley Research Center, Hampton, VA 23681-2199
}

\begin{abstract}
A complete turbulence model, where the turbulent Prandtl and Schmidt numbers are calculated as part of the solution and where averages involving chemical source terms are modeled, is presented. The ability of avoiding the use of assumed or evolution Probability Distribution Functions (PDF's) results in a highly efficient algorithm for reacting flows. The predictions of the model are compared with two sets of experiments involving supersonic mixing and one involving supersonic combustion. The results demonstrate the need for consideration of turbulence/chemistry interactions in supersonic combustion. In general, good agreement with experiment is indicated.
\end{abstract}

\section{Introduction}

Accurate prediction of flows in scramjet engines requires the development of turbulence models that calculate the turbulent Prandtl, $P r_{t}$, and Schmidt, $S c_{t}$, numbers as part of the solution, and account for turbulence/chemistry interactions. Traditional turbulence models that only address velocity fluctuations have no mechanism for incorporating turbulence/chemistry interaction and require the specification of both $P r_{t}$ and $S c_{t}$. Such numbers have a profound influence on flow predictions: a low value of $S c_{t}$ can result in engine unstart, while a higher value may result in flame blow-out. ${ }^{1}$ On the other hand, $P r_{t}$ has an important effect on mixing at high speed flows. It is shown in Ref. 2, which considered the role of variable turbulent Schmidt number on the mixing of supersonic streams, that a value of $P r_{t}=0.9$ gave the best fit for data from the experiment of Cutler et al, ${ }^{3}$ while a value of 0.5 gave the best fit for the experiment of Burrows and Kurkov. ${ }^{4}$

In an attempt to address this problem, a series of step-by-step investigations were carried out to develop a model that calculates $P r_{t}$ and $S c_{t}$ as part of the solution and addresses turbulence/chemistry interactions. Thus, in Ref. 2 the role of variable $S c_{t}$ on supersonic mixing was considered, while in Ref. 5 the role of variable $P r_{t}$ on heat flux in the presence of shock wave/boundary interactions was examined. In a more recent investigation, ${ }^{6}$ the variable $S c_{t}$ formulation of Ref. 2 was extended to address reacting flows while assuming a fixed $\mathrm{Pr}_{t}$.

The turbulence/chemistry interaction in Ref. 6 was studied using the multi-variate $\beta$-PDF for mass fractions developed by Girimaji. ${ }^{7}$ A comparison of assumed and evolution PDF's in flows involving supersonic combustion by Baurle et $a l^{8}$ showed that both formulation yielded comparable mean flow predictions. However, assumed PDF's were unable to predicted higher order correlations, such as terms involving chemical production source terms, with any reasonable accuracy. Similar results were encountered in Ref. 6 . It is shown there that the use of Girimaji's PDF has a highly dissipative effect on the concentration variance resulting in poor agreement with experiment. Computations employing evolution PDF's are time consuming and require excessive storage. Because of this, all terms involving production terms are modeled in this work.

\footnotetext{
${ }^{*}$ Research Assistant Professor, Mechanical and Aerospacing Engineering, Member AIAA

${ }^{\dagger}$ Professor, Mechanical and Aerospace Engineering, Fellow AIAA

¥Aerospace Engineer, Hypersonic Airbreathing Propulsion Branch, Senior Member AIAA
} 
The model is used to predict the flows in two sets of mixing experiments, ${ }^{3,4}$ and the reacting experiment of Ref. 4. In general, good agreement is indicated.

\section{Formulation of Problem}

\section{A. Governing Equations}

A variable $P r_{t}$ and $S c_{t}$ formulation requires equations for the variance of enthalpy and its dissipation rate, and the variance of concentrations and its dissipation rate. These equations were derived in Ref. 2 and Ref. 5 for non-reacting flows. The formulation of Ref. 2 was extended in Ref. 6 to reacting flows while keeping $\operatorname{Pr}_{t}$ constant.

The approach that has been used to derive the final set of equations for variable $P r_{t}$ and $S c_{t}$ in the presence of reactions follows the same procedure used in Refs. 2, 5, 6 and 9. This entails deriving the exact equations that govern the variances of concentrations and enthalpy and their dissipation rates from the Navier-Stokes equations and model the resulting equations term by term. This insures that relevant physics is incorporated into the model. Dimensional and tensorial consistency, Galilean invariance, coordinate system independence, and absence of wall or damping function characterize the resulting set of equations which are given in the Appendix.

\section{B. Turbulence/Chemistry Interaction}

The equation for mass fraction variance, $\sigma_{Y}$, contains the term:

$$
\overline{Y_{m}^{\prime \prime} \dot{\omega}_{m}}
$$

where $Y_{m}^{\prime \prime}$ is the fluctuation of the mass fraction of species $m$, and $\dot{\omega}_{m}$ is its mass production rate. Similarly, the equation that governs the enthalpy variance contains the term

$$
\overline{h^{\prime \prime} \dot{\omega}_{m} \Delta h_{f, m}}
$$

where $h^{\prime \prime}$ is the enthalpy fluctuation and $\Delta h_{f, m}$ is the heat of formation of species $m$.

Traditionally, the above terms are evaluated by using an assumed or evolution PDF's or ignored completely. The assumed PDF's are usually a product of Girimaji's multi-variate $\beta$-PDF for mass fraction fluctuations and a Maxwellian for temperature fluctuations. Comparisons of the predictions of assumed and evolution PDF's have been conducted by Baurle et $a l^{8}$ on supersonic combustion of parallel stream. It was shown there that both formulations give comparable mean values. However, assumed PDF's were unable to produce the correct values of the higher order correlations. In particular, they gave the wrong sign for correlations involving mass production rates. This is why, in the absence of evolution PDF's, better predictions are obtained when correlations involving mass production rates are set to zero.

As will be shown, in the Results and Discussion section below, setting correlations involving mass production rate to zero is not an option for the current formulation. Because evolution PDF's require an excessive amount of time and storage, these terms are modeled here. Thus,

$$
2 \sum_{m} \overline{Y_{m}^{\prime \prime} \dot{\omega}_{m}}=C_{Y, 8} \sum_{m} \sqrt{\widetilde{Y_{m}^{\prime \prime 2}}} \overline{\dot{\omega}}_{m}
$$

and

$$
\sum_{m} \overline{h^{\prime \prime} \dot{\omega}_{m} \Delta h_{f, m}}=C_{h, 12} \sqrt{\widetilde{h^{\prime \prime 2}}} \sum_{m} \overline{\dot{\omega}_{m}} \Delta h_{f, m}
$$

\section{Numerical Procedure}

A modification of REACTMB, ${ }^{10}$ a code that has been under development at North Carolina State University over the last several years, is employed in this investigation. It is a general purpose parallel Navier-Stokes solver for multi-component multi-phase reactive flows at all speeds. It employs a second order essentially non-oscillatory (ENO) upwind method based on Low Diffusion Flux Splitting Scheme of Edwards ${ }^{11}$ to discretize the inviscid fluxes while central differences are employed for the viscous and diffusion terms. Planar relaxation is employed and the code is parallelized using domain decomposition and message passing(MPI) strategies. 


\section{Model Constants}

The model constants developed in Refs. 2, 5 and 6 remain unchanged. The final set of model constants are summarized in Table 1 for concentration variance and its dissipation rate, and in Table 2 for enthalpy variance and its dissipation rate.

Table 1. Model constants for $\sigma_{Y}$ and $\epsilon_{Y}$ equations

\begin{tabular}{|c|c|c|c|c|c|c|c|c|c|c|c|c|}
\hline$C_{Y}$ & $C_{Y, 1}$ & $C_{Y, 2}$ & $C_{Y, 3}$ & $C_{Y, 41}$ & $C_{Y, 42}$ & $C_{Y, 5}$ & $C_{Y, 6}$ & $C_{Y, 7}$ & $C_{Y, 8}$ & $C_{Y, p}$ & $C_{Y, 9}$ & $\sigma_{h}$ \\
\hline 0.065 & 1.0 & 0.095 & -0.025 & 0.45 & -1.0 & 1.0 & 0.5 & 0.78125 & 0.25 & -0.1 & 1.0 & 0.5 \\
\hline
\end{tabular}

Table 2. Model constants for $\widetilde{h^{\prime \prime 2}}$ and $\epsilon_{h}$ equations

\begin{tabular}{|c|c|c|c|c|c|c|c|c|c|c|}
\hline$C_{h}$ & $C_{h, 2}$ & $C_{h, 4}$ & $C_{h, 5}$ & $C_{h, 6}$ & $C_{h, 7}$ & $C_{h, 8}$ & $C_{h, 9}$ & $C_{h, 10}$ & $C_{h, 11}$ & $C_{h, 12}$ \\
\hline 0.0648 & 0.5 & -0.4 & -0.04 & -0.12 & 1.45 & 0.7597 & 0.87 & 0.25 & -1.5 & -0.75 \\
\hline
\end{tabular}

\section{Results and Discussion}

A theory that is developed to predict $P r_{t}$ and $S c_{t}$ as part of the solution should apply for both reacting and non-reacting flows. Because of this, the present theory is validated by two sets of experiments involving supersonic mixing, ${ }^{3,4}$ and one experiment involving supersonic combustion. ${ }^{4}$ In the experiment of Cutler et $a l,{ }^{3}$ a coaxial nozzle was designed to produce two uniform coaxial jets at exit. The center jet consists of $95 \%$ of $\mathrm{He}$ and $5 \% \mathrm{O}_{2}$ by volume at a Mach number $M=1.8$, while the outer jet is air at $M=1.8$. A schematic of the experiment is shown in Fig. 1. The grids employed are the ones used in Refs. 2 and 3. The fine grid consists of 188, 080 cells and is decomposed into 13 blocks for parallel computing while the intermediate grid deletes every other point in the axial direction. All results employed here model the flow in the nozzle, employ the fine grid and use the axisymmetric version of REACTMB.

The second set of experiments are those of Burrows and Kurkov. ${ }^{4}$ A schematic of the experiment is shown in Fig. 2. Hydrogen is injected into the test section through a nickel injector parallel to the vitiated main flow. The mixing case employed nitrogen in place of air for the main flow. At the entrance of the test section, $M=2.44$, the static pressure is one atmosphere, and the static temperature is in the range 1250-1270 K for the reacting case, and about $1150 \mathrm{~K}$ for the mixing case. In both cases, hydrogen was injected at $M=1$, matched pressure and a total temperature slightly above the ambient temperature. The two grids that are employed here are those used in Ref. 6. Each grid consists of 15 blocks. The first grid has 86,643 cells, while the second has 104,428 cells. The fine grid reflects grid refinement in the blocks where mixing of the two streams takes place. Rather than using measured conditions at the inlet of the test section, the flows in both hydrogen and nitrogen/air nozzle were computed. It was necessary to iterate on inflow conditions of both nozzles to arrive at the stipulated conditions at the exit of each nozzle. All results presented here employed the fine grid.

In Ref. 2, calculations of Cutler et al experiments ${ }^{3}$ employed a variable $S c_{t}$ and a $P r_{t}=0.9$. Results are presented in Figs.3-5, which compare predictions of current theory at selected stations with those of Ref.2 and the experiment. As is seen from Fig. 3, the results of Ref. 2 for mass fraction of $\mathrm{He}$ at $x=261 \mathrm{~mm}$ are better than the current prediction. Figures 4 and 5 show that predictions for velocity and Pitot pressure are comparable.

Figure 6 shows a comparison of mass fraction prediction with the mixing experiment of Ref. 4 . As is seen from the figure, good agreement is indicated. Similar results were obtained in Ref. 6 .

Two sets of figures are presented for the reacting case of Ref. 4. For this calculation, the seven-species, seven-reaction $\mathrm{H}_{2}$-Air model used in Ref. 6 which was originally developed by Jachimowski ${ }^{12}$ is employed. In the first, terms involving averages of chemical source terms are ignored, while in the second, the contributions of these terms are included. As is seen from Fig. 7, poor agreement with the experiment is indicated. Figures 8 and 9 show contours of $S c_{t}$ and $P r_{t}$. It appears that the main cause of the discrepancy is a result of a reduced $\mathrm{Pr}_{t}$ near the mixing region. This has the tendency of promoting heat transfer and early combustion. Figure 
10 shows that when the contributions of the chemical source terms are included, much better agreement with the experiment is indicated. Figures 11 and 12 show contours of $S c_{t}$ and $P r_{t}$, respectively.

Based on the above, two relevant observations can be made. The first is that modeling of averages of terms involving chemical source terms is a viable option. When this approach is compared with approaches requiring assumed or evolution PDF's, a great deal of computational efficiency is achieved. Second, a relatively inexpensive calculation of variable $P r_{t}$ and $S c_{t}$ can be obtained by assuming a value for the Lewis number, and eliminating either the equations for enthalpy variance and its dissipation rate, or those for the variance of concentrations and its dissipation rate. This, however, will result in ignoring one of the averages involving chemical source terms. Because inclusion of such terms is important, assuming a constant Lewis number is not recommended.

\section{Conclusions}

A complete turbulence model where both $P r_{t}$ and $S c_{t}$ are calculated as part of the solution, and where averages of terms involving chemical source terms are modeled is presented. Thus, calculations of turbulent flow become similar to those of laminar flows in the sense that all that is required is to specify initial and boundary conditions.

Because the resulting algorithm does not require the use of an assumed or evolution PDF, it is computationally efficient, especially when one deals with complex three-dimensional geometries characteristics of proposed scramjet designs.

The resulting algorithm, which is based on the exact Navier-Stokes equations, is dimensionally and tensorially consistent, Galilean invariant, coordinate system independent and free of damping and wall functions. Although, the algorithm is applied to relatively simple geometries, past experiences suggest that such an approach works well for complicated geometry without having to adjust any of the model constants.

Finally, averages involving chemical source terms are important and shall always be included in combustion calculations.

\section{Acknowledgment}

We wish to express our appreciation to Mr. George Rumford, program manager of the Defense Test Resource Management Center's(DTRMC) Test and Evaluation/Science and Technology(T\&E/S\&T) program, for funding this effort under the Hypersonic Test focus area.

\section{References}

${ }^{1}$ Eklund, D. R., Baurle, R. A., and Gruber, M. R., "Numerical Study of a Scramjet Combustor Fueled by an Aerodynamic Ramp Injector in Dual-Mode Combustion," AIAA Paper 2001-0379, January 2001.

${ }^{2}$ Xiao, X., Edwards, J. R., Hassan, H. A., and Cutler, A. D., "Variable Turbulent Schmidt Number Formulation for Scramjet Applications," AIAA Paper 2005-1099, January 2005, To appear in AIAA Journal.

${ }^{3}$ Cutler, A. D., Carty, A. A., Doemer, S. E., Diskin, G. S., and Drummond, J. P., "Supersonic Coaxial Jet Flow Experiment for CFD Validation," AIAA Paper 1999-3388, July 1999.

${ }^{4}$ Burrows, M. C. and Kurkov, A. P., "Analytical and Experimental Study of Supersonic Combustion of Hydrogen in a Vitiated Airstream," NASA TM X-2828, September 1973.

${ }^{5}$ Xiao, X., Edwards, J. R., Hassan, H. A., and Gaffney Jr, R. L., "Role of Turbulent Prandtl Number on Heat Flux at Hypersonic Mach Numbers," AIAA Paper 2005-1098, January 2005.

${ }^{6}$ Keistler, P. G., Gaffney Jr, R. L. J., Xiao, X., and Hassan, H. A., "Turbulence Modeling for Scramjet Applications," AIAA Paper 2005-5382, June 2005.

${ }^{7}$ Girimaji, S. S., "Simple Recipe for Modeling Reaction-Rates in Flows with Turbulent-Combustion," AIAA Paper 19911792, June 1991

${ }^{8}$ Baurle, R. A., Hsu, A. T., and Hassan, H. A., "Assumed and Evolution Probability Density Functions in Supersonic Turbulent Combustion Calculations," Journal of Propulsion and Power, Vol. 11, No. 6, 1995, pp. 1132-1138.

${ }^{9}$ Robinson, D. F. and Hassan, H. A., "Further Development of the $k-\zeta$ (Enstrophy) Turbulence Closure Model," AIAA Journal, Vol. 36, No. 10, 1998, pp. 1825-1833.

${ }^{10}$ Edwards, J. R., "Advanced Implicit Algorithm for Hydrogen-Air Combustion Calculation," AIAA Paper 1996-3129, June 1996.

${ }^{11}$ Edwards, J. R., "A Low Diffusion Flux Splitting Scheme for Navier-Stokes Calculations," Computers 83 Fluids, Vol. 26, No. 6, 1997, pp. 635-659.

${ }^{12}$ Jachimowski, C. J., "An Analytic Study of the Hydrogen-Air Reaction Mechanism with Application to Scramjet Combustion," NASA Technical Paper 2791, February 1988. 


\section{A. Model Equations}

The Favre-averaged species conservation equations can be written as

$$
\frac{\partial}{\partial t}\left(\bar{\rho} \widetilde{Y}_{m}\right)+\frac{\partial}{\partial x_{j}}\left(\bar{\rho} \tilde{u}_{j} \widetilde{Y}_{m}\right)=\frac{\partial}{\partial x_{j}}\left(\bar{\rho} D \frac{\partial \widetilde{Y}_{m}}{\partial x_{j}}-\bar{\rho} \widetilde{Y_{m}^{\prime \prime} u_{j}^{\prime \prime}}\right)+\overline{\dot{\omega}_{m}}
$$

where

$$
-\bar{\rho} \widetilde{Y_{m}^{\prime \prime} u_{j}^{\prime \prime}}=\bar{\rho} D_{t} \frac{\partial \widetilde{Y}_{m}}{\partial x_{j}},
$$

$\bar{\rho}$ is the density, $\widetilde{Y}_{m}$ is the mass fraction of species $m, \tilde{u}_{i}$ is the velocity, $D$ is the laminar diffusion coefficient and $D_{t}$ is the turbulent diffusion coefficient.

The variance of mass fractions, $\sigma_{Y}$, is defined as

$$
\sigma_{Y}=\sum_{m} \widetilde{Y_{m}^{\prime \prime 2}}
$$

while its dissipation rate, $\epsilon_{Y}$, is defined as

$$
\bar{\rho} \epsilon_{Y}=\overline{\sum_{m} \rho D\left(\frac{\partial Y_{m}^{\prime \prime}}{\partial x_{j}}\right)^{2}}
$$

The $\sigma_{Y^{-}}$equation is given as

$$
\begin{aligned}
\frac{\partial}{\partial t}\left(\bar{\rho} \sigma_{Y}\right)+\frac{\partial}{\partial x_{j}}\left(\bar{\rho} \tilde{u}_{j} \sigma_{Y}\right)= & \frac{\partial}{\partial x_{j}}\left[\bar{\rho}\left(D+C_{Y, 1} D_{t}\right) \frac{\partial \sigma_{Y}}{\partial x_{j}}\right] \\
& +2 \sum_{m} \bar{\rho} D_{t}\left(\frac{\partial \widetilde{Y}_{m}}{\partial x_{j}}\right)^{2}-2 \bar{\rho} \epsilon_{Y}+2 \sum_{m} \overline{\dot{\omega}_{m} Y_{m}^{\prime \prime}} \\
& +C_{Y, p} \frac{\bar{\rho}}{\bar{P} \tau_{Y}} \max \left(\frac{D \bar{P}}{D t}, 0.0\right)
\end{aligned}
$$

where

$$
\begin{gathered}
\tau_{Y}=\frac{\sigma_{Y}}{\epsilon_{Y}} \\
D_{t}=\frac{1}{2}\left(C_{Y} \frac{k \sigma_{Y}}{\epsilon_{Y}}+\frac{\nu_{t}}{\sigma_{h}}\right), \quad k=\frac{1}{2} \widetilde{u_{i}^{\prime \prime} u_{i}^{\prime \prime}}, \quad \nu_{t}=C_{\mu} \frac{k^{2}}{\nu \zeta} \\
2 \sum_{m} \overline{\bar{\omega}_{m} Y_{m}^{\prime \prime}}=C_{Y, 8} \sum_{m} \sqrt{\widetilde{Y_{m}^{\prime \prime}}} \frac{\dot{\omega}_{m}}{}
\end{gathered}
$$

$\bar{P}$ is the pressure, $\nu_{t}$ is the turbulent kinematic viscosity, $k$ is the turbulent kinetic energy and $\zeta$ is the enstrophy.

The $\epsilon_{Y}$-equation is given as

$$
\begin{aligned}
\frac{\partial}{\partial t}\left(\bar{\rho} \epsilon_{Y}\right)+\frac{\partial}{\partial x_{j}}\left(\bar{\rho} \tilde{u}_{j} \epsilon_{Y}\right)= & \frac{\partial}{\partial x_{j}}\left[\bar{\rho}\left(D+C_{Y, 5} D_{t}\right) \frac{\partial \epsilon_{Y}}{\partial x_{j}}\right] \\
& +2 \bar{\rho} \epsilon_{Y}\left(\frac{1}{3} \frac{\partial \tilde{u}_{i}}{\partial x_{i}}+C_{Y, 2} b_{j k} \frac{\partial \tilde{u}_{j}}{\partial x_{k}}\right)+C_{Y, 3} \bar{\rho} k \sum_{m} \frac{\partial}{\partial x_{j}} \sqrt{\widetilde{Y_{m}^{\prime \prime 2}}} \frac{\partial \widetilde{Y}_{m}}{\partial x_{j}} \\
& +\bar{\rho} D C_{Y, 41} D_{t} \sum_{m}\left(\frac{\partial^{2} \widetilde{Y}_{m}}{\partial x_{j} \partial x_{j}}\right)^{2}+\bar{\rho} D \frac{C_{Y, 42}}{\tau_{Y}} \sum_{m} \sqrt{\widetilde{Y_{m}^{\prime \prime 2}}} \frac{\partial^{2}}{\partial x_{k} \partial x_{k}} \\
& \bar{\rho} D_{t} \frac{C_{Y, 6}}{\tau_{Y}} \sum_{m}\left(\frac{\partial \widetilde{Y}}{\partial x_{j}}\right)^{2}-C_{Y, 7} \bar{\rho} \frac{\epsilon_{Y}}{\tau_{Y}}+\frac{C_{Y, 9}}{\tau_{Y}} \sum_{m} \sqrt{\widetilde{Y_{m}^{\prime \prime}}} \frac{\dot{\omega}_{m}}{}
\end{aligned}
$$


where

$$
b_{j k}=\frac{\tau_{j k}}{\bar{\rho} k}+\frac{2}{3} \delta_{j k}, \quad \tau_{j k}=-\overline{\rho u_{j}^{\prime \prime} u_{k}^{\prime \prime}}
$$

The turbulent Schmidt number is defined as

$$
S c_{t}=\frac{\nu_{t}}{D_{t}}
$$

The mean energy equation can be written as

$$
\frac{\partial}{\partial t}(\bar{\rho} \tilde{h})+\frac{\partial}{\partial x_{j}}\left(\bar{\rho} \tilde{u}_{j} \tilde{h}\right)=\frac{D \bar{P}}{D t}-\frac{\partial \bar{q}_{i}}{\partial x_{i}}+\bar{\phi}-\frac{\partial}{\partial x_{j}}\left(\overline{\rho h^{\prime \prime} u_{j}^{\prime \prime}}\right)
$$

where

$$
\begin{aligned}
\bar{q}_{i} & =-\left(\lambda \frac{\partial \tilde{T}}{\partial x_{i}}+\bar{\rho} D \sum_{m} \tilde{h}_{m} \frac{\partial \tilde{Y}_{m}}{\partial x_{i}}\right) \\
-\overline{\rho h^{\prime \prime} u_{j}^{\prime \prime}} & \equiv q_{t, j}=\bar{\rho}\left(\alpha_{t} \frac{\partial \tilde{h}}{\partial x_{j}}+D_{t} \sum_{m} \tilde{h}_{m} \frac{\partial \tilde{Y}_{m}}{\partial x_{j}}\right) \\
\bar{\phi} & =\bar{t}_{i j} \frac{\partial \tilde{u}_{i}}{\partial x_{j}}+\bar{\rho} \epsilon, \quad \epsilon=\nu \zeta \\
\bar{t}_{i j} & =2 \mu\left(S_{i j}-\frac{1}{3} \delta_{i j} \frac{\partial \tilde{u}_{k}}{\partial x_{k}}\right), \quad S_{i j}=\frac{1}{2}\left(\frac{\partial \tilde{u}_{i}}{\partial x_{j}}+\frac{\partial \tilde{u}_{j}}{\partial x_{i}}\right)
\end{aligned}
$$

$\tilde{h}$ is the enthalpy, $\bar{q}_{i}$ is the laminar heat flux, and $\alpha_{t}$ is the turbulent diffusivity.

The enthalpy variance $\left(\widetilde{h^{\prime \prime 2}}\right)$ equation can be written as

$$
\begin{aligned}
\frac{\partial}{\partial t}\left(\bar{\rho} \widetilde{h^{\prime \prime 2}} / 2\right)+\frac{\partial}{\partial x_{j}}\left(\bar{\rho} \tilde{u}_{j} \widetilde{h^{\prime \prime 2}} / 2\right)= & \frac{\partial}{\partial x_{j}}\left[\bar{\rho}\left(\gamma \alpha+\alpha_{t} C_{h, 2}\right) \frac{\partial \widetilde{h^{\prime \prime 2}}}{\partial x_{j}}\right] \\
& +2 \mu \gamma S_{i j}\left[\frac{\partial}{\partial x_{j}}\left(\frac{q_{t, i}}{\bar{\rho}}\right)+\frac{\partial}{\partial x_{i}}\left(\frac{q_{t, j}}{\bar{\rho}}\right)\right]-\frac{4}{3} \mu \gamma S_{k k} \frac{\partial}{\partial x_{j}}\left(\frac{q_{t, j}}{\bar{\rho}}\right) \\
& -(\gamma-1) \bar{\rho} \widetilde{h^{\prime \prime 2}} \frac{\partial \tilde{u}_{i}}{\partial x_{i}}-q_{t, i} \frac{\partial \tilde{h}}{\partial x_{i}}+2 C_{h, 4} \gamma \mu \sqrt{\widetilde{h^{\prime \prime 2}}} \zeta-\gamma \bar{\rho} \epsilon_{h} \\
& -\sum_{m} \overline{h^{\prime \prime} \dot{\omega}_{m}} \Delta h_{f, m}
\end{aligned}
$$

with

$$
\begin{aligned}
\gamma & =C_{p} / C_{v} \\
\alpha_{t} & =\frac{1}{2}\left(C_{h} k \tau_{h}+\frac{\nu_{t}}{0.89}\right), \\
\tau_{h} & =\frac{\overline{h^{\prime \prime 2}}}{\epsilon_{h}} \\
\epsilon_{h} & =\alpha\left(\frac{\partial h^{\prime \prime}}{\partial x_{i}}\right)^{2} \\
\sum_{m} \overline{h^{\prime \prime} \dot{\omega}_{m}} \Delta h_{f, m} & =C_{h, 12} \sqrt{\widetilde{h^{\prime \prime 2}}} \sum_{m} \frac{\dot{\omega}_{m}}{h_{f, m}},
\end{aligned}
$$

where $\epsilon_{h}$ is the dissipation rate of the enthalpy variance, and $\alpha$ is the laminar diffusivity. 
The equation for the dissipation rate of enthalpy variance is taken as

$$
\begin{aligned}
\frac{\partial}{\partial t}\left(\bar{\rho} \epsilon_{h}\right)+\frac{\partial}{\partial x_{j}}\left(\bar{\rho} \tilde{u}_{j} \epsilon_{h}\right)= & -\bar{\rho} \epsilon_{h}\left(C_{h, 5} b_{j k}-\frac{\delta_{j k}}{3}\right) \frac{\partial \tilde{u}_{j}}{\partial x_{k}} \\
& +C_{h, 6} \bar{\rho} k \frac{\partial \sqrt{h^{\prime \prime 2}}}{\partial x_{j}} \frac{\partial \tilde{h}}{\partial x_{j}}+\frac{\partial}{\partial x_{j}}\left[\left(\gamma \alpha+C_{h, 7} \alpha_{t}\right) \frac{\partial \epsilon_{h}}{\partial x_{j}}\right] \\
& C_{h, 8} \frac{q_{t, j}}{\tau_{h}} \frac{\partial \tilde{h}}{\partial x_{j}}-\gamma \bar{\rho} \epsilon_{h}\left(\frac{C_{h, 9}}{\tau_{h}}+\frac{C_{h, 10}}{\tau_{k}}\right) \\
& +C_{h, 11} \epsilon_{h}\left[\frac{D \bar{\rho}}{D t}+\frac{\bar{\rho}}{\bar{P}} \max \left(\frac{D \bar{P}}{D t}, 0.0\right)\right]
\end{aligned}
$$

where

$$
\tau_{k}=\frac{k}{\nu \zeta}
$$

The model constants, $C_{h}$ and $C_{h, 1-12}$, are given in Table 2. The turbulent $P r_{t}$ is defined as

$$
\operatorname{Pr}_{t}=\frac{\nu_{t}}{\alpha_{t}}
$$

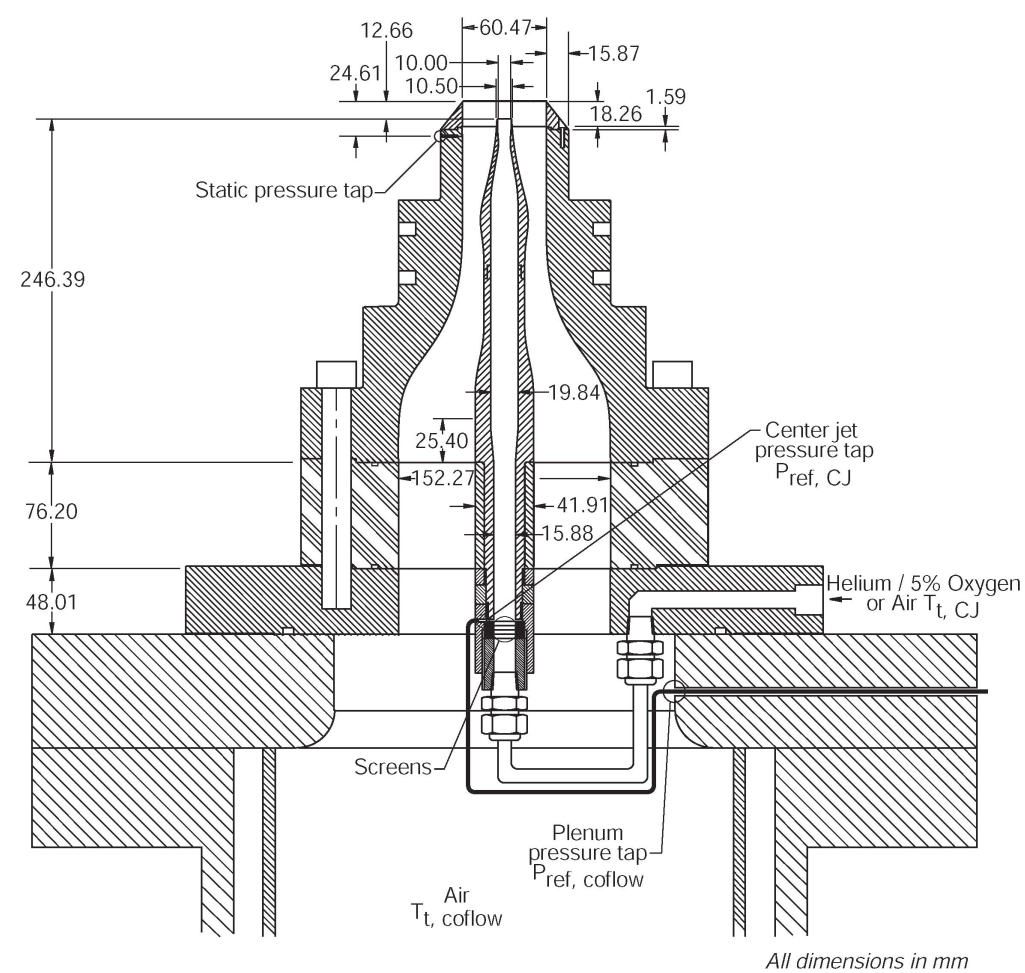

Figure 1. Schematic of Experiment Setup (Ref. 3) 


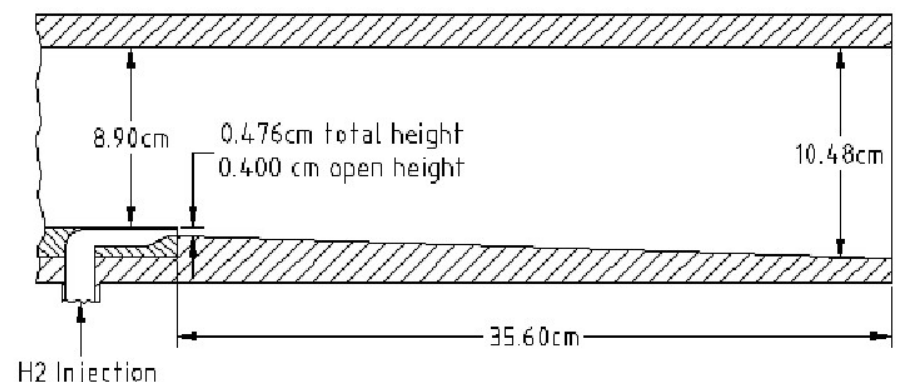

Figure 2. Schematic of Experiment Setup (Ref. 4)

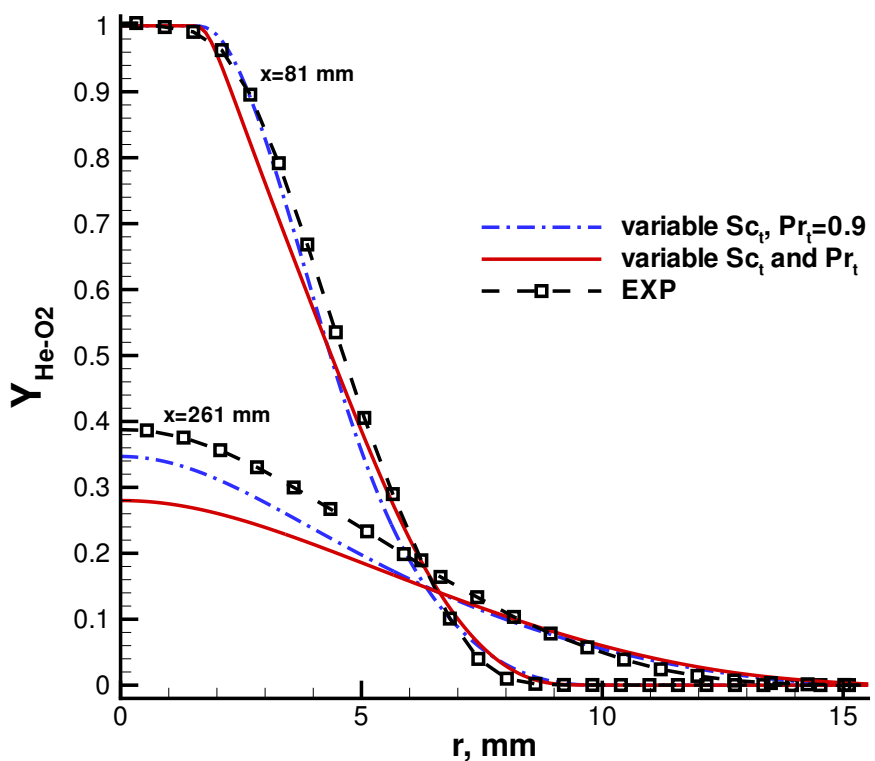

Figure 3. Comparison of computed and measured $\mathrm{He}_{-} \mathrm{O}_{2}$ mass fraction(Ref. 3) 


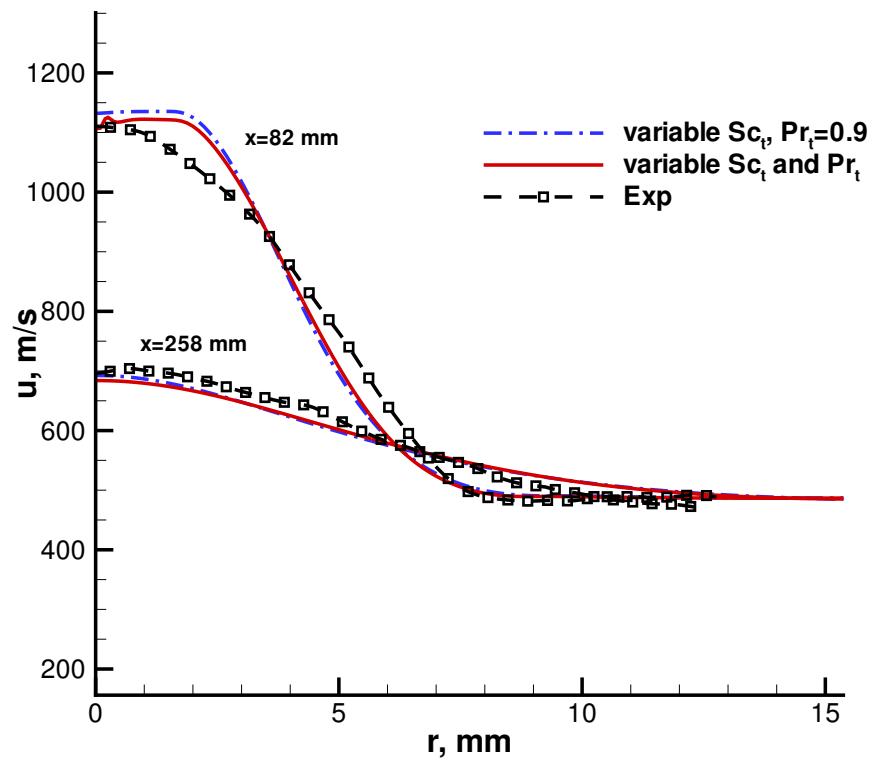

Figure 4. Comparison of computed and measured velocities (Ref. 3)

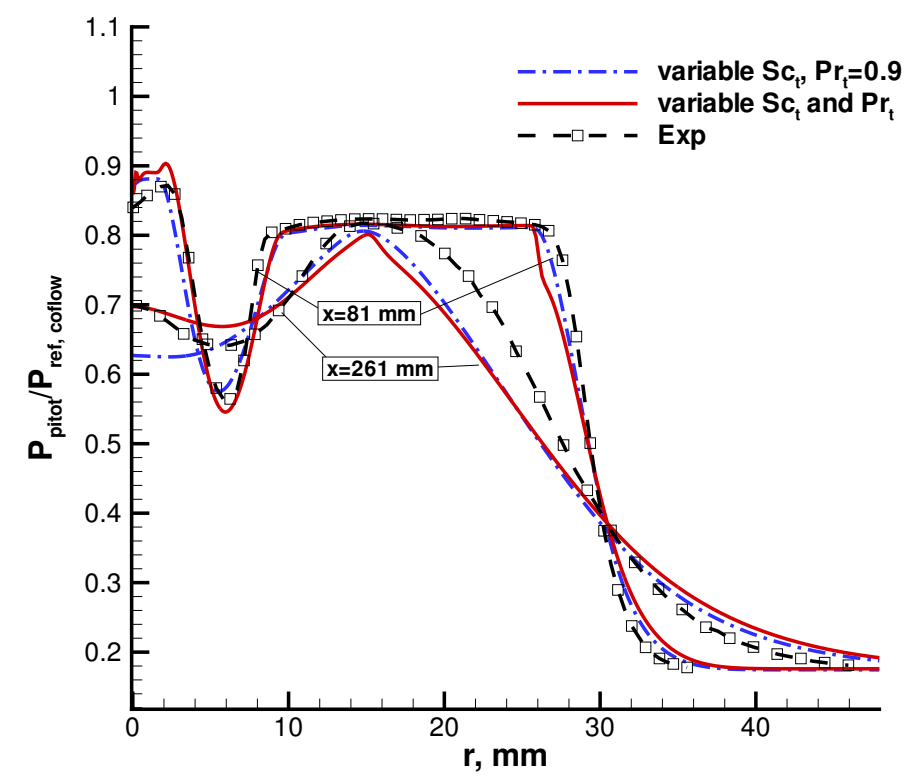

Figure 5. Comparison of computed and measured Pitot pressure (Ref. 3) 


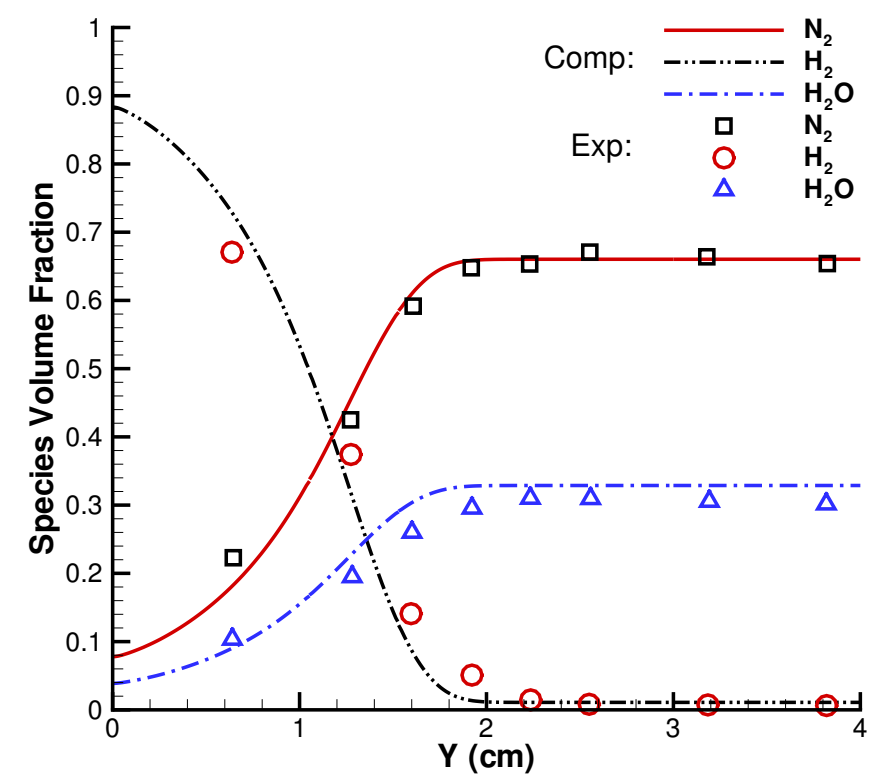

Figure 6. Comparison of computed and measured volume fractions (Ref. 4), mixing case

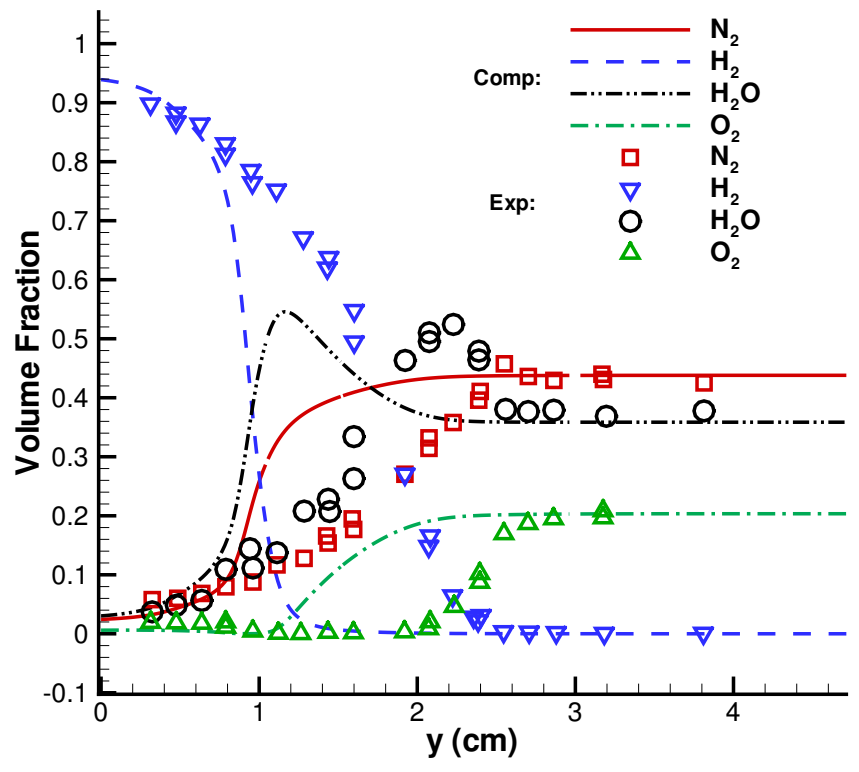

Figure 7. Comparison of computed and measured volume fractions (Ref. 4), reacting case, without chemical source terms 


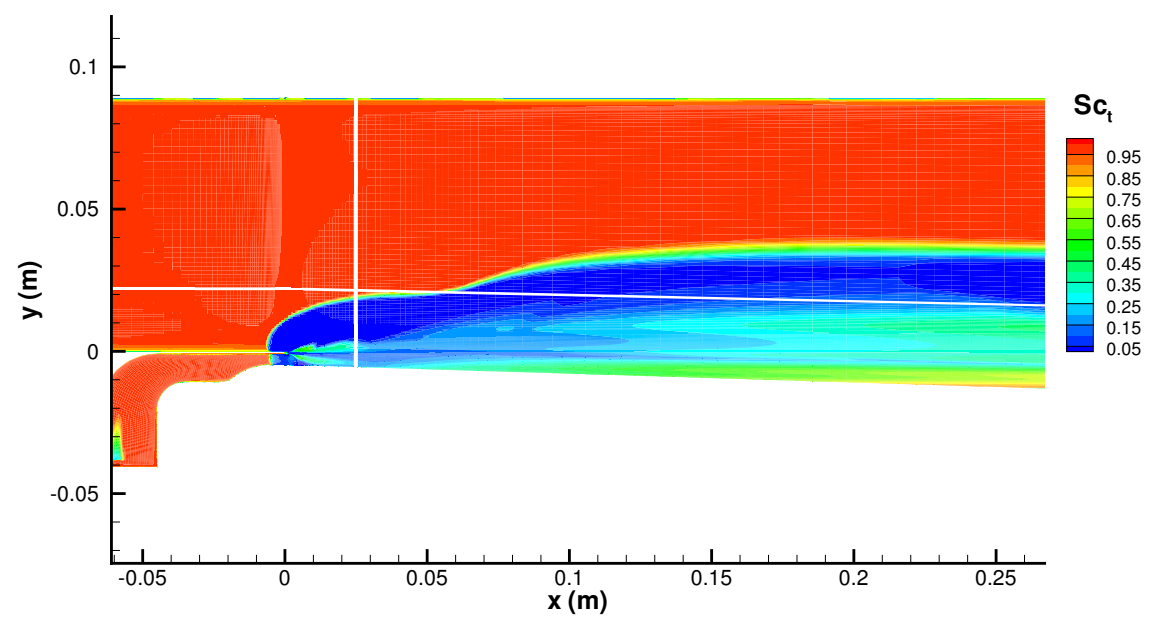

Figure 8. Schmidt number contours, reacting case, without chemical source terms

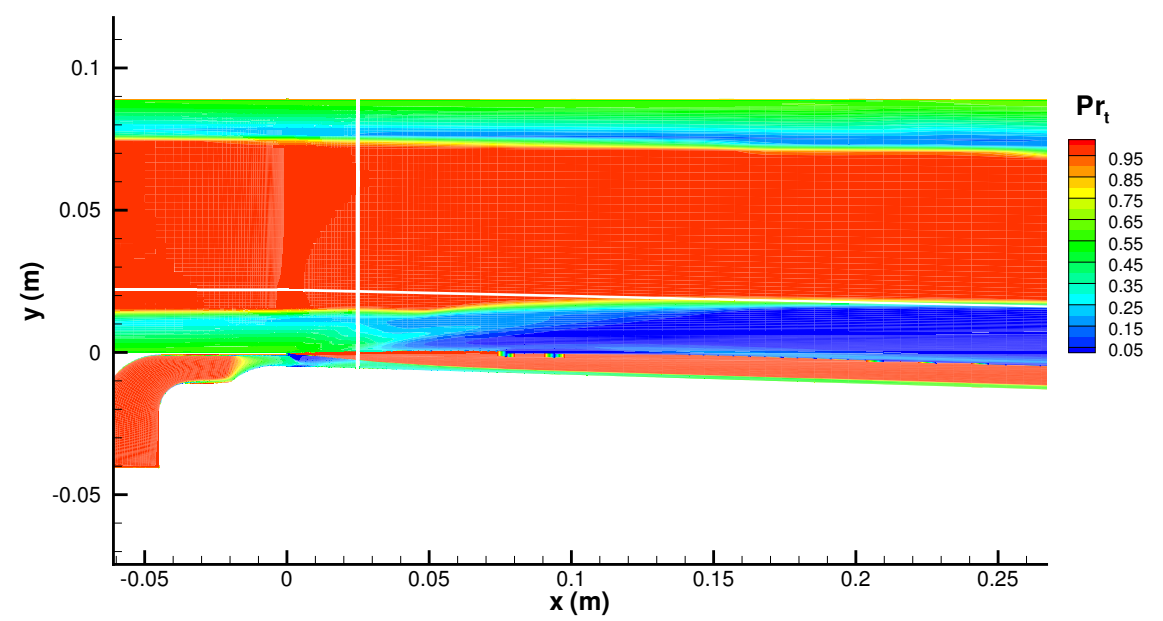

Figure 9. Prandtl number contours, reacting case, without chemical source terms 


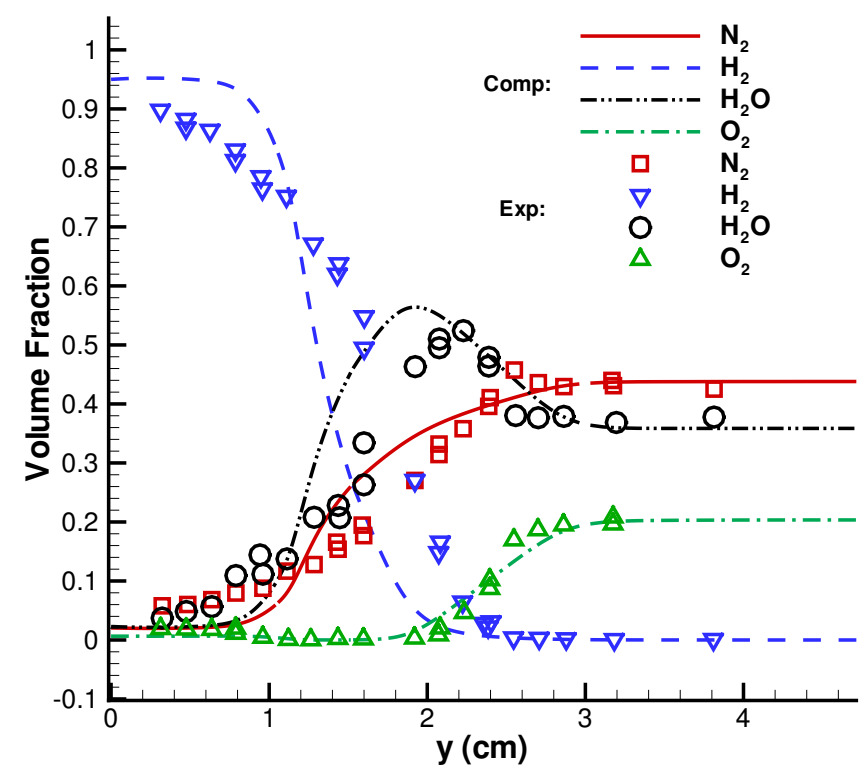

Figure 10. Comparison of computed and measured volume fractions (Ref. 4), reacting case, with chemical source terms

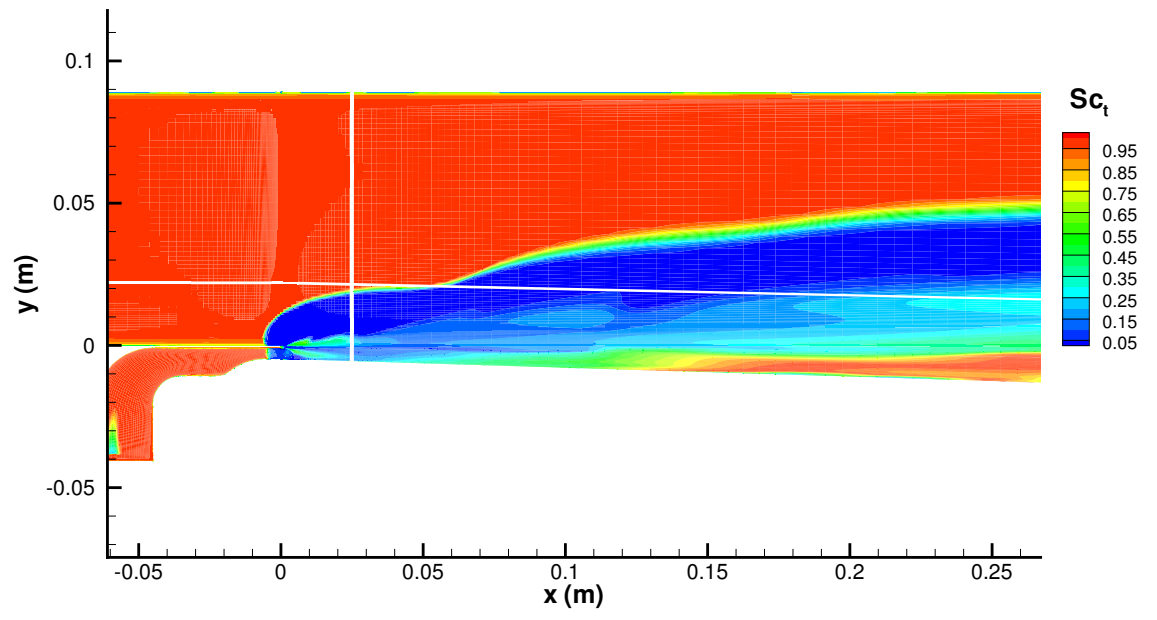

Figure 11. Schmidt number contours, reacting case, with chemical source term 


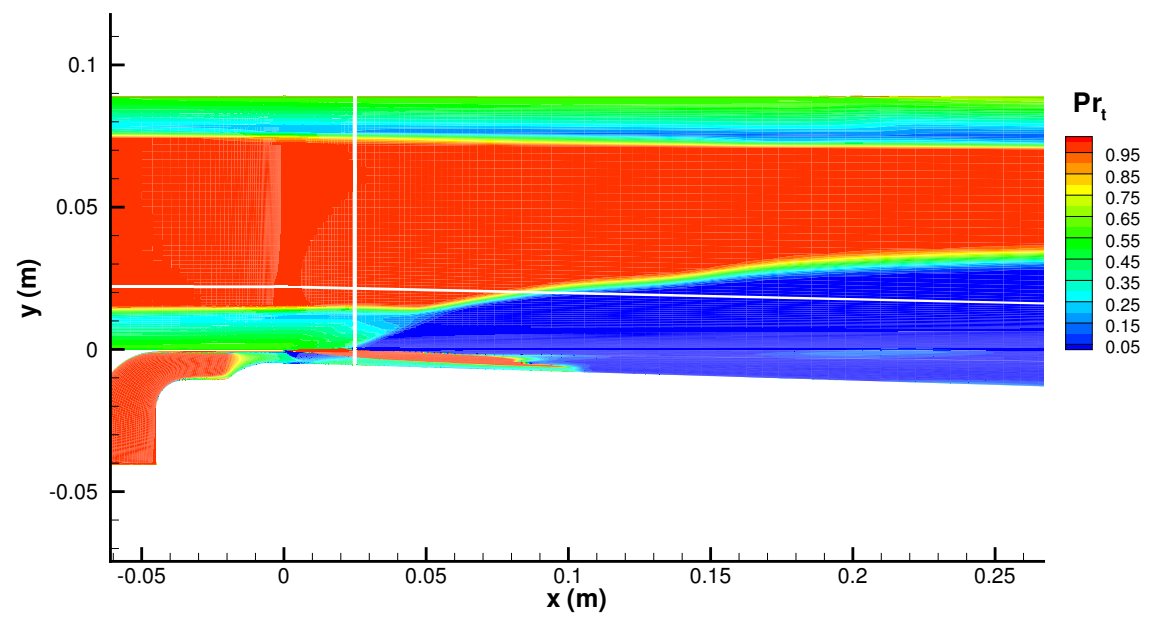

Figure 12. Prandtl number contours, reacting case, with chemical source term 